\title{
Schwarzschild Like Solution with Global Monopole in Bumblebee Gravity
}

\author{
İbrahim Güllü* and Ali Övgün ${ }^{\dagger}$ \\ Physics Department, Eastern Mediterranean University, \\ Famagusta, 99628 North Cyprus, via Mersin 10, Turkey
}

\begin{abstract}
In this paper, by considering Einstein-Hilbert-Bumblebee (EHB) gravity around global monopole field, we derive exactly a black hole spacetime metric. To test the effect of global monopole field and bumblebee field, which causes the spontaneous Lorentz symmetry breaking, we calculate the weak deflection angle using the Gauss-Bonnet theorem.
\end{abstract}

\section{INTRODUCTION}

Monopoles which are formed by gauge-symmetry breaking during the phase transitions in the early universe can be the source of inflation $[1,2]$. On the other hand, global monopoles result from a global symmetry braking of global $O(3)$ symmetry into $U(1)$ in phase transitions in the universe. The effect of these monopoles on Schwarzschild spacetime are discussed in [3] by analyzing the particle orbit and Hawking radiation. On the other side, the Lorentz symmetry breaking is associated with the idea that the Quantum Gravity (QG) signals may emerge at low energy scales [4]. The naturelness and the possibility of Lorentz symmetry breaking is discussed in the context of string theory in [5-15]. The Lorentz symmety breaking arises in other theories like noncomutative field theories [16-18] and loop quantum gravity theory [19, 20] among other scenarios [21-24].

Einstein's theory of general relativity, which is a metric theory of gravitation [25], has successfully passed through many experimental tests. One of the important testing technique is a gravitational lensing which helps us to understand galaxies, dark matter, dark energy and the universe [26-28]. This technique firstly was used by Eddington [29], afterwards, various works on gravitational lensing have been done for black holes, wormholes, global monopoles and other objects [30-35]. There are many method to calculate gravitational lensing [36-40]. Recently, new method is derived by Gibbons and Werner which the deflection angle of light can be calculated from non-rotating asymptotically flat spacetimes using the Gauss-Bonnet theorem on the optical geometry of the black hole [41], then it is extended to stationary spacetimes by Werner [42]. Since then, many works have been done, one can see [43]-[92].

The aim of the manuscript is to obtain a Schwarzschild like solution with global monopole of Einstein equation in the presence of spontaneous Lorentz symmetry breaking. We then study the effect of global monopoles and spontaneous Lorentz symmetry breaking on the deflection of light. We also discuss the effect of global monopoles on Lorentz symmetry breaking.

The manuscript is organized as follows: In section II, we derive the Einstein fields equations for Einstein-HilbertBumblebee gravity around a global monopole fields. In section III, we obtain new Schwarzschild-like black hole solution for Einstein-Hilbert-Bumblebee (EHB) gravity around a global monopole. Section IV is devoted to computation of the weak deflection angle by Schwarzschild-like black hole solution for EHB gravity around a global monopole using the GBT. We conclude our results in section V.

\section{EHB GRAVITY AROUND A GLOBAL MONOPOLE}

In this part we review the EHB gravity [4] whose Lagrangian density is introduced as follows

$\mathrm{L}_{B}=\sqrt{-g}\left(\frac{1}{2 \kappa} R+\frac{\xi}{2 \kappa} B^{\mu} B^{\nu} R_{\mu \nu}-\frac{1}{4} B_{\mu \nu}^{2}-V\left(B^{\mu} B_{\mu}\right)\right)+\mathcal{L}_{M}$, where $B^{\mu}$ is the bumblebee field with field strength tensor $B_{\mu \nu}=\partial_{\mu} B_{\nu}-\partial_{\nu} B_{\mu}, V\left(B_{\mu}\right)$ being the potential which has terms responsible for the spontaneous Lorentz symmetry breaking and $\xi$ is the coupling constant of nonminimal gravity-bumblebee interaction. $\mathcal{L}_{M}$ is the Lagrangian density of matter, which in our case is the global monopole, and others field contents with their couplings to the bumblebee field. The equations of motion of (II) is $\mathrm{G}_{\mu \nu}=\kappa\left(T_{\mu \nu}^{B}+T_{\mu \nu}^{M}\right)$ after varying (II) with respect to the metric $g_{\mu \nu}$. We defined the Einstein tensor $\mathcal{G}_{\mu \nu}=R_{\mu \nu}-\frac{1}{2} g_{\mu \nu} R$ and energy-momentum tensor $T_{\mu \nu}=T_{\mu \nu}^{B}+T_{\mu \nu}^{M}$ where $T_{\mu \nu}^{B}$ is the contribution of the bumblebee field to the energy-momentum tensor $\mathrm{T}_{\mu \nu}^{B} \equiv-B_{\mu \sigma} B^{\sigma}{ }_{\nu}-\frac{1}{4} g_{\mu \nu} B_{\alpha \beta}^{2}-$

\footnotetext{
*Electronic address: ibrahim.gullu@emu.edu.tr

${ }^{\dagger}$ Electronic address: ali.ovgun@emu.edu.tr; https://www.aovgun.com
} 
$g_{\mu \nu} V\left(B^{\mu} B_{\mu}\right)+4 V^{\prime} B_{\mu} B_{\nu}$ $+\frac{\xi}{\kappa}\left(\frac{1}{2} g_{\mu \nu} B^{\alpha} B^{\beta} R_{\alpha \beta}-B_{\nu} B^{\alpha} R_{\alpha \mu}-B_{\mu} B^{\alpha} R_{\alpha \nu}\right)$

$+\frac{\xi}{\kappa}\left(\frac{1}{2} \nabla_{\alpha} \nabla_{\mu}\left(B^{\alpha} B_{\nu}\right)+\frac{1}{2} \nabla_{\alpha} \nabla_{\nu}\left(B_{\mu} B^{\alpha}\right)\right)$

$+\frac{\xi}{\kappa}\left(-\frac{1}{2} \nabla^{\lambda} \nabla_{\lambda}\left(B_{\mu} B_{\nu}\right)-\frac{1}{2} g_{\mu \nu} \nabla_{\alpha} \nabla_{\beta}\left(B^{\alpha} B^{\beta}\right)\right)$, and $T_{\mu \nu}^{M}$ is the matter sector of the energy-momentum tensor which is defined as $\mathrm{T}_{\mu}^{(M) \nu}=\operatorname{diag}\left(\frac{\eta^{2}}{r^{2}}, \frac{\eta^{2}}{r^{2}}, 0,0\right)$ where $\eta$ is the constant term which is related to the global monopole charge. Note that the total energy-momentum tensor is covariantly conserved. $\nabla^{\mu} T_{\mu \nu}=0$. Taking the variation of (II) with respect to the bumblebee field $B_{\mu}$ provides the second equation of motion

$$
\nabla^{\mu} B_{\mu \nu}=J_{\nu}
$$

where $J_{\nu}=J_{\nu}^{B}+J_{\nu}^{M}$, with $J_{\nu}^{M}$ acting as a source term for the bumblebee field and $J_{\nu}^{B}=2 V^{\prime} B_{\nu}-\frac{\xi}{\kappa} B^{\mu} R_{\mu \nu}$ is the current due to self interaction of the bumblebee field.

The trace of (II) is $-1 \frac{}{\kappa R=-4 V\left(B^{\mu} B_{\mu}\right)+4 V^{\prime} B_{\mu} B^{\mu}+\frac{\xi}{\kappa}\left(-\frac{1}{2} \nabla^{\lambda} \nabla_{\lambda}\left(B_{\mu} B^{\mu}\right)-\nabla_{\alpha} \nabla_{\beta}\left(B^{\alpha} B^{\beta}\right)\right)+T^{M}}$. Inserting the (II) back into (II)

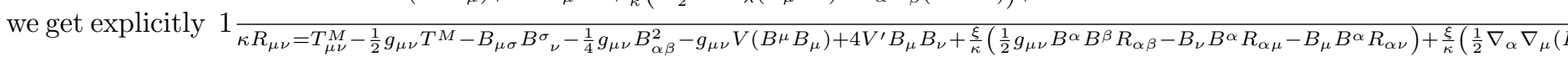

The potential $V\left(B_{\mu} B^{\mu}\right)$ is chosen in a way that the vacuum expectation value (VEV) for $B_{\mu}$ has to be nonvanishing in order to have spontaneous Lorentz symmetry breaking. Because of this reason, the general form of the potential, $V\left(B_{\mu} B^{\mu} \pm b^{2}\right)$ where $b^{2}$ is a real positive constant, is set to zero to determine the VEV of the bumblebee field which gives the following condition $\mathrm{B}_{\mu} B^{\mu} \pm b^{2}=0$. The solution of (II) has a nonnull vacuum expectation value $\left\langle B^{\mu}\right\rangle=b^{\mu}$ which breaks the Lorentz symmetry [4].

\section{THE SCHWARZSCHILD LIKE SOLUTION OF EHB GRAVITY AROUND GLOBAL MONOPOLE}

In this part a static spherically symmetric solution to the Einstein equations is obtained. We take the Birkhoff metric as an ansatz $\mathrm{g}_{\mu \nu}=\operatorname{diag}\left(-e^{2 \gamma}, e^{2 \rho}, r^{2}, r^{2} \sin ^{2} \theta\right)$, where $\gamma$ and $\rho$ are functions of $r$, and fix the Bumblebee field in its vacuum expectation value [93]

$\mathrm{B}_{\mu}=b_{\mu}$, which results in $\mathrm{V}=0, \quad \mathrm{~V}^{\prime}=0$. Also we take

$$
b_{\mu}=\left(0, b_{r}(r), 0,0\right)
$$

for which the field strength vanishes, $b_{\mu \nu}=0$. The condition $b^{\mu} b_{\mu}=b^{2}=$ constant gives the explicit form of the radial background field $\mathrm{b}_{r}(r)=|b| e^{\rho}$. Then, (II) can be written as following $0=\bar{R}_{\mu \nu}$

$=R_{\mu \nu}-\kappa\left(T_{\mu \nu}^{M}-\frac{1}{2} g_{\mu \nu} T^{M}\right)$

$-\frac{\xi}{2} g_{\mu \nu} b^{\alpha} b^{\beta} R_{\alpha \beta}+\xi b_{\nu} b^{\alpha} R_{\alpha \mu}+\xi b_{\mu} b^{\alpha} R_{\alpha \nu}$

$-\frac{\xi}{2} \nabla_{\alpha} \nabla_{\mu}\left(b^{\alpha} b_{\nu}\right)-\frac{\xi}{2} \nabla_{\alpha} \nabla_{\nu}\left(b_{\mu} b^{\alpha}\right)$

$+\frac{\xi}{2} \nabla^{\lambda} \nabla_{\lambda}\left(b_{\mu} b_{\nu}\right)$. where the trace of the energy-momentum tensor is $\mathrm{T}^{M}=2 \frac{\eta^{2}}{r^{2}}$. The combination constructed from the energy-momentum tensor and its trace in (III) reads $\mathrm{T}_{\mu \nu}^{M}-\frac{1}{2} g_{\mu \nu} T^{M}=\left(0,0,-\eta^{2},-\eta^{2} \operatorname{Sin}^{2}(\theta)\right)$, and the components of the Ricci tensor in (III) are $\mathrm{R}_{t t}=e^{2(\gamma-\rho)}\left[\partial_{r}^{2} \gamma+\left(\partial_{r} \gamma\right)^{2}-\partial_{r} \gamma \partial_{r} \rho+\frac{2}{r} \partial_{r} \gamma\right]$,

$R_{r r}=-\partial_{r}^{2} \gamma-\left(\partial_{r} \gamma\right)^{2}+\partial_{r} \gamma \partial_{r} \rho+\frac{2}{r} \partial_{r} \rho$,

$R_{\theta \theta}=e^{-2 \rho}\left[r\left(\partial_{r} \rho-\partial_{r} \gamma\right)-1\right]+1$. The components of (III) become $\bar{R}_{t t}=\left(1+\frac{\ell}{2}\right) R_{t t}+\frac{\ell}{r}\left(\partial_{r} \rho+\partial_{r} \gamma\right) e^{2(\gamma-\rho)}$,

$\bar{R}_{r r}=\left(1+\frac{3 \ell}{2}\right) R_{r r}$,

$\bar{R}_{\theta \theta}=(1+\ell) R_{\theta \theta}-\ell\left(\frac{1}{2} r^{2} e^{-2 \rho} R_{r r}+1\right)+\eta^{2}$,

$\bar{R}_{\phi \phi}=\sin ^{2}(\theta) \bar{R}_{\theta \theta}$, where $\ell=\xi b^{2}$. These equations (III,III,III,III) are indipendently equal to zero. Therefore, the following combination can be written to find the function $\rho(r)[4] \mathrm{r}^{2} e^{-2 \rho} \bar{R}_{t t}+r^{2} e^{-2 \rho} \bar{R}_{r r}+2 \bar{R}_{\theta \theta}=0$, which yields $\mathrm{e}^{2 \rho}=(1+\ell)\left(1+\eta^{2}-\frac{\rho_{0}}{r}\right)^{-1}$. In order to find the function $\gamma(r)$ the following second combination is constructed [4] from (III,III,III,III) which is $\mathrm{r}^{2} e^{-2 \gamma} \bar{R}_{t t}-\left(1+\frac{2}{\ell}\right) \bar{R}_{\theta \theta}=0$. After a straightforward calculation one reach the following function $\mathrm{e}^{2 \gamma}=1+\eta^{2}-\frac{\rho_{0}}{r}$. Then the Lorentz symmetry breaking spherically symmetric solution for EHB can be written as $\mathrm{ds}^{2}=-\left(1-\mu-\frac{2 M}{r}\right) d t^{2}+(1+\ell)\left(1-\mu-\frac{2 M}{r}\right)^{-1} d r^{2}$

$+r^{2} d \theta^{2}+r^{2} \sin ^{2} \theta d \phi^{2}$ where we have defined $\rho_{0}=2 M$ and the global monopole term $\bar{\mu}$ which is defined as $\bar{\mu}=-\eta^{2}$. The metric (III) recovers LSB spherically symmetric solution when $\eta=0$ [4] and the usual Schwarzschild metric for limit $\ell \rightarrow 0$. 


\section{WEAK DEFLECTION ANGLE OF SCHWARZSCHILD LIKE SOLUTION WITH GLOBAL MONOPOLE IN EHB GRAVITY}

First, we introduce the following coordinate transformation: $r \rightarrow(1-\bar{\mu})^{-1 / 2} r, t \rightarrow(1-\bar{\mu})^{1 / 2} t, M \rightarrow$ $(1-\bar{\mu})^{-3 / 2} M$ : The spacetime metric of the Lorentz symmetry breaking spherically symmetric solution for EHB becomes: $\quad \mathrm{ds}^{2}=-\left(1-\frac{2 M}{r}\right) d t^{2}+(1+\ell)\left(1-\frac{2 M}{r}\right)^{-1} d r^{2}+g^{2} r^{2}\left(d \theta^{2}+\sin ^{2} \theta d \phi^{2}\right)$ where $g^{2}=1-\bar{\mu}$. To obtain the weak deflection angle of Schwarzschild Like Solution with Global Monopole in Bumblebee Gravity, we write the metric in the optical form within equatorial plane $\theta=\pi / 2$, and obtain null geodesics $\left(\mathrm{d} s^{2}=0\right)$ :

$$
\mathrm{d} t^{2}=\frac{(1+\ell) \mathrm{d} r^{2}}{f(r)^{2}}+\frac{g^{2} r^{2} \mathrm{~d} \varphi^{2}}{f(r)}
$$

where $f(r)=\left(1-\frac{2 M}{r}\right)$. Afterwards, we calculate the Gaussian optical curvature $K$ in 2 -dimensions for the above space, which gives an intrinsic property of the space:

$$
K=\frac{R_{\text {icciScalar }}}{2} \approx-2 \frac{M}{r^{3}}+2 \frac{M l}{r^{3}}-2 \frac{M l^{2}}{r^{3}} .
$$

Note, that the Gaussian optical curvature is found as negative in leading order terms, which imply that all the light rays locally diverge. Hence, after converging, to obtain the multiple images, we will use the Gauss-Bonnet theorem for the region $D_{R}$ in $M$, with boundary $\partial D_{R}=\gamma_{\tilde{g}} \cup C_{R}[41]$

$$
\begin{gathered}
D_{R} K \mathrm{~d} S+\oint_{\partial D_{R}} \kappa \mathrm{d} t=2 \pi \chi\left(D_{R}\right)-\left(\theta_{O}+\theta_{S}\right)=\pi . \\
D_{R} K \mathrm{~d} S+\oint_{\partial D_{R}} \kappa \mathrm{d} t=\pi,
\end{gathered}
$$

where the $\kappa$ stands for the geodesics curvature. Moreover, $\theta_{O}+\theta_{S} \rightarrow \pi$ implies that jump angles become $\pi / 2$ when $R$ going to infinity. Also one can say that $D_{R}$ is non-singular region, so that the Euler characteristic is $\chi\left(D_{R}\right)=1$. Then, $\kappa\left(\gamma_{\tilde{g}}\right)=0$. In addition, the near asymptotic limit of $R, C_{R}:=r(\varphi)=R=$ const., one can write the radial component of the geodesic curvature as follows: [41]

$$
\kappa\left(C_{R}\right)=\left|\nabla_{\dot{C}_{R}} \dot{C}_{R}\right|=\left(\tilde{g}_{r r} \dot{C}_{R}^{r} \dot{C}_{R}^{r}\right)^{\frac{1}{2}} \rightarrow \frac{-1}{R}
$$

After substitute this result, yielding: $\kappa\left(C_{R}\right) \mathrm{d} t=\frac{g}{\sqrt{(1+\ell)}} \mathrm{d} \varphi$. Using the straight line approximation for the weak field regions, $r_{s}=u / \sin \varphi$ at zeroth-order, where $u$ is the impact parameter. Then the Gauss-Bonnet equation becomes:

$$
\pi=\int_{0}^{\pi} \int_{\frac{u}{\sin \varphi}}^{\infty} K \mathrm{~d} S+\frac{g}{\sqrt{(}(1+\ell)} \int_{0}^{\pi+\alpha} \mathrm{d} \varphi
$$

where optical surface area is defined as $\mathrm{d} S=r \mathrm{~d} r \mathrm{~d} \varphi$, and $\alpha$ is a deflection angle.

Afterwards, the optical geometry curvature within the Gauss-Bonnet theorem def1, give us the weak deflection angle:

$$
\alpha=\frac{\sqrt{(1+\ell) \pi}}{g}-\pi-\sqrt{(1+\ell)} \int_{0}^{\pi} \int_{\frac{u}{\sin \varphi}}^{\infty} K r \mathrm{~d} r \mathrm{~d} \varphi .
$$

Hence, the weak deflection angle $\alpha$ of Schwarzschild Like Solution with Global Monopole in Bumblebee Gravity in weak field limits is found as follows:

$$
\alpha \simeq 4 \frac{M}{u}+\frac{l \pi}{2}+2 \frac{M l}{u}+\frac{\bar{\mu} \pi}{2} .
$$

Note that the bumblebee parameter $\ell$, the mass term and the global monopole term $\bar{\mu}$ all of them increase the deflection angle. The expression of weak deflection angle is consistent with [92] when global monopole term $\bar{\mu}=0$. 


\section{CONCLUSIONS}

We have investigated a static spherically symmetric vacuum solution for the EHB gravity in the presence of a global monopole. We have obtained a spherically symmetric solution similar to the Schwarzschild black hole. This solution reduces to spherically symmetric solution in the LSB scenario when $\bar{\mu}=0$. We have also calculated the deflection angle of the light in this geometry by using the GBT. It is found that both the bumblebee parameter and the global monopole term increases the deflection angle. If the global monopole term is set to $\bar{\mu}=-\ell$ then part of the effect of the bumblebee parameter on the deflection angle cancel out. However, the bumblebee parameter still gives a correction to the deflection angle. In addition to this, setting $\bar{\mu}=-\ell\left(1+\frac{4 M}{u \pi}\right)$ directly remove the effect of the bumblebee parameter on the deflection angle.

[1] J. P. Preskill, Phys. Rev. Lett. 43, 1365 (1979).

[2] M. Barriola and A. Vilenkin, Phys. Rev. Lett. 63, 341 (1989).

[3] N. Dadhich, K. Narayan, and U. A. Yajnik, Pramana - J Phys 50, 307-314 (1998).

[4] R. Casana, A. Cavalcante, F. P. Poulis, and E. B. Santos, Phys. Rev. D 97, 104001 (2018).

[5] V. A. and S. Samuel, Phys. Rev. D 39, 683 (1989).

[6] V. A. Kostelecký and S. Samuel, Phys. Rev. Lett. 63, 224 (1989).

[7] V. A. Kostelecký and S. Samuel, Phys. Rev. D 40, 1886 (1989).

[8] V. A. and R. Potting, Nucl. Phys. B 359, 545 (1991).

[9] R. V. Maluf and J. C. S. Neves, [arXiv:2011.12841 [gr-qc]].

[10] C. Ding and X. Chen, [arXiv:2008.10474 [gr-qc]].

[11] S. Chen, M. Wang and J. Jing, JHEP 07, 054 (2020)

[12] A. Ali and K. Saifullah, [arXiv:2004.02005 [gr-qc]].

[13] W. D. R. Jesus and A. F. Santos, Int. J. Mod. Phys. A 35, no.09, 2050050 (2020)

[14] S. Kanzi and İ. Sakallı, Nucl. Phys. B 946, 114703 (2019)

[15] R. Oliveira, D. M. Dantas, V. Santos and C. A. S. Almeida, Class. Quant. Grav. 36, no.10, 105013 (2019)

[16] S. M. Carroll, J. A. Harvey, V. A. Kostelecky, C. D. Lane, and T. Okamoto, Phys. Rev. Lett. 87, 141601 (2001).

[17] I. Mocioiu, M. Pospelov, and R. Roiban, Phys. Lett. B 489, 390 (2000).

[18] A. F. Ferrari, M. Gomes, J. R. Nascimento, E. Passos, A. Yu. Petrov, and A. J. da Silva, Phys. Lett. B 652, 174 (2007).

[19] R. Gambini and J. Pullin, Phys. Rev. D 59, 124021 (1999).

[20] J. R. Ellis, N. E. Mavromatos, and D. V. Nanopoulos, Gen. Relativ. Gravit. 32, 127 (2000).

[21] M. S. Berger and V. A. Kostelecký, Phys. Rev. D 65, 091701 (2002).

[22] D. Blas and S. Sibiryakov, J. Exp. Theor. Phys. 120, 509 (2015).

[23] R. Lehnert, Hyperfine Interact. 215, 25 (2013).

[24] D. S. Gorbunov and S. M. Sibiryakov, J. High Energy Phys. 09, 082 (2005).

[25] A. Einstein, Annalen Phys. 49, no.7, 769-822 (1916).

[26] B. P. Abbott et al. [LIGO Scientific and Virgo], Phys. Rev. Lett. 116, no.6, 061102 (2016).

[27] K. Akiyama et al. [Event Horizon Telescope], Astrophys. J. 875, no.1, L1 (2019).

[28] M. Bartelmann and P. Schneider, Phys. Rept. 340, 291 (2001).

[29] F. W. Dyson, A. S. Eddington and C. Davidson, Phil. Trans. Roy. Soc. Lond. A 220, 291-333 (1920).

[30] K.S. Virbhadra and G. F. R. Ellis, Phys. Rev. D 62, 084003 (2000).

[31] C. R. Keeton, C. S. Kochanek and E. E. Falco, Astrophys. J. 509, 561-578 (1998).

[32] V. Bozza, Phys. Rev. D 66, 103001 (2002).

[33] V. Bozza, Gen. Rel. Grav. 42, 2269-2300 (2010).

[34] S. b. Chen and J. l. Jing, Phys. Rev. D 80, 024036 (2009).

[35] E. F. Eiroa, G. E. Romero and D. F. Torres, Phys. Rev. D 66, 024010 (2002).

[36] M. Sharif and S. Iftikhar, Astrophys. Space Sci. 357, no.1, 85 (2015).

[37] W. G. Cao and Y. Xie, Eur. Phys. J. C 78, no.3, 191 (2018).

[38] G. S. Bisnovatyi-Kogan and O. Y. Tsupko, Universe 3, no.3, 57 (2017).

[39] G. S. Bisnovatyi-Kogan and O. Y. Tsupko, Mon. Not. Roy. Astron. Soc. 404, 1790-1800 (2010).

[40] P. V. P. Cunha and C. A. R. Herdeiro, Gen. Rel. Grav. 50, no.4, 42 (2018).

[41] G. W. Gibbons and M. C. Werner, Class. Quant. Grav. 25, 235009 (2008).

[42] M. C. Werner, Gen. Rel. Grav. 44, 3047-3057 (2012).

[43] G. Crisnejo and E. Gallo, Phys. Rev. D 97, no.12, 124016 (2018).

[44] K. Jusufi, I. Sakalli, and A. Övgün, Phys. Rev. D 96, no.2, 024040 (2017).

[45] A. Övgün, K. Jusufi, and I. Sakalli, Annals Phys. 399, 193-203 (2018).

[46] K. Jusufi and A. Övgün, Phys. Rev. D 97, no.6, 064030 (2018).

[47] K. Jusufi, M. C. Werner, A. Banerjee, and A. Övgün, Phys. Rev. D 95, no.10, 104012 (2017).

[48] A. Övgün, Phys. Rev. D 99, no.10, 104075 (2019). 
[49] W. Javed, R. Babar, and A. Övgün, Phys. Rev. D 100, no.10, 104032 (2019).

[50] W. Javed, J. Abbas, A. Övgün, Eur. Phys. J. C 79, no.8, 694 (2019).

[51] I. Sakalli and A. Övgün, EPL 118, no.6, 60006 (2017).

[52] K. de Leon and I. Vega, Phys. Rev. D 99, no.12, 124007 (2019).

[53] Z. Li and T. Zhou, Phys. Rev. D 101, no.4, 044043 (2020).

[54] A. Övgün, I. Sakalli, and J. Saavedra, Annals Phys. 411, 167978 (2019).

[55] A. Övgün, I. Sakalli, and J. Saavedra, JCAP 10, 041 (2018).

[56] K. Jusufi and A. Övgün, Int. J. Geom. Meth. Mod. Phys. 16, no. 08, 1950116 (2019).

[57] Y. Kumaran and A. Övgün, Chin. Phys. C 44, no.2, 025101 (2020).

[58] R. Kumar, S. G. Ghosh and A. Wang, Phys. Rev. D 101, no.10, 104001 (2020).

[59] W. Javed, J. Abbas and A. Övgün, Phys. Rev. D 100, no.4, 044052 (2019).

[60] W. Javed, A. Hazma and A. Övgün, Phys. Rev. D 101, no.10, 103521 (2020).

[61] A. Ishihara, Y. Suzuki, T. Ono and H. Asada, Phys. Rev. D 95, no.4, 044017 (2017).

[62] T. Ono, A. Ishihara and H. Asada, Phys. Rev. D 96, no.10, 104037 (2017).

[63] T. Ono, A. Ishihara and H. Asada, Phys. Rev. D 99, no.12, 124030 (2019).

[64] T. Ono and H. Asada, Universe 5, no.11, 218 (2019).

[65] H. Arakida, Gen. Rel. Grav. 50, no.5, 48 (2018).

[66] Z. Li and A. Övgün, Phys. Rev. D 101, no.2, 024040 (2020).

[67] Z. Li, G. Zhang and A. Övgün, Phys. Rev. D 101, no.12, 124058 (2020).

[68] W. Javed, M. B. Khadim, A. Övgün and J. Abbas, Eur. Phys. J. Plus 135, no.3, 314 (2020).

[69] W. Javed, M. B. Khadim and A. Övgün, Eur. Phys. J. Plus 135, 595 (2020).

[70] Z. Li and J. Jia, Eur. Phys. J. C 80, no.2, 157 (2020).

[71] Z. Li and T. Zhou, '[arXiv:2001.01642 [gr-qc]].

[72] K. Takizawa, T. Ono and H. Asada, Phys. Rev. D 101, no.10, 104032 (2020).

[73] G. W. Gibbons, Class. Quant. Grav. 33, no.2, 025004 (2016).

[74] S. U. Islam, R. Kumar and S. G. Ghosh, JCAP 09, 030 (2020).

[75] R. C. Pantig and E. T. Rodulfo, Chin. J. Phys. 66, 691-702 (2020).

[76] N. Tsukamoto, Phys. Rev. D 101, no.10, 104021 (2020).

[77] K. Jusufi, A. Övgün, and A. Banerjee, Phys. Rev. D 96, no.8, 084036 (2017).

[78] A. Övgün, Turk. J. Phys. 44, 465-471 (2020).

[79] A. Övgün and İ. Sakalli, Class. Quant. Grav. 37, no.22, 225003 (2020).

[80] A. Belhaj, M. Benali, A. E. Balali, H. El Moumni and S. E. Ennadifi, Class. Quant. Grav. 37, no.21, 215004 (2020).

[81] K. Jusufi, A. Övgün, J. Saavedra, Y. Vasquez, and P. A. Gonzalez, Phys. Rev. D 97, no.12, 124024 (2018).

[82] A. Övgün, K. Jusufi, and I. Sakalli, Phys. Rev. D 99, no.2, 024042 (2019).

[83] W. Javed, R. Babar, and A. Övgün, Phys. Rev. D 99, no.8, 084012 (2019).

[84] K. Jusufi and A. Övgün, Phys. Rev. D 97, no.2, 024042 (2018).

[85] K. Jusufi, A. Övgün, A. Banerjee and I. Sakalli, Eur. Phys. J. Plus 134, no.9, 428 (2019).

[86] A. Övgün, G. Gyulchev, and K. Jusufi, Annals Phys. 406, 152-172 (2019).

[87] A. Övgün, Universe 5, 115 (2019).

[88] A. Övgün, Phys. Rev. D 98, no.4, 044033 (2018).

[89] Z. Li, G. He and T. Zhou, Phys. Rev. D 101, no.4, 044001 (2020).

[90] P. Goulart, Class. Quant. Grav. 35, no.2, 025012 (2018).

[91] T. Ono, A. Ishihara and H. Asada, Phys. Rev. D 98, no.4, 044047 (2018).

[92] Z. Li and A. Övgün, Phys. Rev. D 101, no.2, 024040 (2020).

[93] O. Bertolami and J. Paramos, Phys. Rev. D 72, 044001 (2005). 